\title{
Abstracts of papers presented at the 11th Mammalian Molecular and Biochemical Genetics Workshop meeting held at the Linnean Society Rooms, Picadilly, London on 27 and 28 November 1984
}

\section{Studies on the splicing of $\mathbf{H - 2}$ genes}

\author{
By ALAN L. ARCHIBALD AND SUNE KVIST \\ European Molecular Biology Laboratory, Postfach 10.2209 Meyerhofstrasse 1, \\ D-6900 Heidelberg, FRG
}

The murine $H-2 K^{k}$ and $K^{b}$ genes are $90 \%$ homologous at the nucleotide level. This homology is particularly marked at the $3^{\prime}$ end where from the end of exon 5 to the termination codon there are only two nucleotide differences, both in the last intron. These differences, however, appear to cause a difference in the splicing of the last exon. Site directed mutagenesis has been used to study the effect of these base changes on splicing. The mutated genes were introduced into mouse 1T22.6 cells and their expression was monitored by $\mathrm{S} 1$ mapping and also by immunoprecipitation and SDS-PAGE analysis. The effect of the mutations on the splicing of the mRNA and consequent size of the antigen will be discussed.

\section{Crystallin gene expression in chick development and transdifferentiation}

\author{
BY D. JANE BOWER AND L. H. ERRINGTON \\ University of Edinburgh, Genetics Department, King's Buildings, West Mains \\ Road, Edinburgh EH9 $3 J N$
}

The highly conserved, major proteins of the vertebrate lens belong to four families, the $\alpha, \beta, \gamma$ and $\delta$ crystallins. cDNA and genomic clones corresponding to chick $\alpha, \beta$ and $\delta$ crystallin genes have been isolated and used to study transcription of these genes in lens development, and in in vitro transdifferentiation of tissue to lens cells. Extralenticular transcription of some of the genes in vivo has also been detected and compared with expression in lens tissue. 


\title{
Microdissection and microcloning of the mouse X-chromosome
}

\author{
By S. D. M. BROWN, E. FISHER AND J. S. CAVANNA \\ Department of Biochemistry, St. Mary's Hospital Medical School, Paddington, \\ London W2 1PG,
}

Microdissection and microcloning techniques are being used to obtain genomic sequences from the mouse $X$-chromosome. From one microdissection of a hundred whole $X$-chromosomes, a total of 2000 genomic clones were recovered. Restriction analysis of 39 clones demonstrated an average insert size of $1.2 \mathrm{~Kb}$, the size of inserts contained repeat sequences as revealed by hybridization to total mouse DNA. A further microdissection from a 100 chromosomes of a region proximal to the centre of the $\mathrm{X}$-chromosome and encompassing the $m d x$ locus provided 550 genomic clones. Such regional dissections provide banks of clones premapped to an individual region of the chromosome and will greatly aid in the molecular mapping and investigation of individual chromosomal regions.

\section{Identification of a cDNA clone coding for $T c p$ - 1 , a $t$-locus specific protein}

\author{
BY KEITH DUDLEY, KEITH R. WILLISON, JEAN POTTER AND \\ MARY F. LYON* \\ Institute of Cancer Research, Chester Beatty Laboratories, Fulham Road, London \\ SW3 6JB, and *MRC Radiobiology Unit, Harwell, Oxon OX11 ORD
}

The $t$-locus of the mouse occupies a 15 centimorgan portion of the proximal end of chromosome 17 and influences embryonic development and male fertility in a manner which has yet to be described on a molecular basis. We have made a cDNA library from mRNA isolated from the testes of wild type mice and, using Northern blotting, have selected clones which are expressed at high levels in post-meiotic cells. Because of the way the $t$-locus influences male fertility and in particular transmission distortion, these clones are good candidates for $t$-locus encoded sequences. Using Southern blots of DNA from wild type and $t$-mutant mice we have identified a clone, B1 .4, which showsa polymorphism on $t$-DNA. Partial $t$-haplotypes have allowed us to map this clone to the region of chromosome 17 corresponding to the locus of the $t$-specific protein $T c p-1^{a}$. This blotting data has been supported by using hybrid selected translation to show that clone B1.4 will select an mRNA from RNA isolated from the testes of wild type mice which encodes a protein having a pI slightly more basic than the protein translated from hybrid selected $t$-mutant RNA. 


\title{
Introduction of human phosphoglycerate kinase (PGK) cDNA into a PGK-deficient line of Chinese hamster ovary cells
}

\author{
By PELIN FAIK, STEPHEN RAWSON, JAMES H. WALKER AND \\ MICHAEL J. MORGAN \\ Department of Biochemistry, University of Leicester, Leicester LE1 $7 R H$
}

\begin{abstract}
A full length copy of the coding sequence of human PGK was introduced into a PGK deficient line of CHO-K1 cells by DNA-mediated gene transfer by: (a) calcium phosphate-DNA coprecipitate or $(b)$ by fusion of bacterial protoplasts containing the PGK encoded in a plasmid. Transformants were isolated (i) by cotransfer of the antibiotic resistance marker, AGPT, which confers selectable resistance to the antibiotic G418 (genticin); or (ii) by a direct selection for expression of the cDNA clone. The PGK-deficient cell line is unable to metabolize mannose, and indeed mannose is toxic. Restoration of PGK activity should restore the ability to grow on mannose and/or limit toxicity. Both methods of DMGT enabled PGK positive transformants to be isolated and these have been shown to behave as would be predicted from the wild type, i.e. they now catabolize mannose to lactic acid and mannose is not toxic. Expression of the human PGK has been demonstrated by starch gel electrophoresis of the enzyme and by Southern blot analysis.
\end{abstract}

\section{Concentration of pyruvate kinase isoenzymes in the liver of mutant phenotypes in the mouse}

\author{
BY LESLEY FITTON AND GRAHAME BULFIELD
}

Genetics Group, AFRC's Poultry Research Centre, Roslin, Midlothian EH25 9 PS

Pyruvate kinase (PK) has a complex pattern of tissue specific regulation with 4 isoenzymes and $4 \mathrm{mRNAs}$ from only two (unlinked) structural genes. PK isoenzymes are also developmentally regulated in the liver: $\mathrm{L}$ (coded for by $P k-1$ ) replacing the foetal $\mathrm{M}_{2}$ (coded for by $P k-3$ ) in adults.

We have several putative regulatory gene mutations recovered from laboratory and feral mice which affect the activity of liver PK in adult mice. We have now used specific anti-L and anti- $M_{2}$ sera to titrate the concentration of the $L$ and $M_{2}$ isoenzyme in these mutant phenotypes. 


\title{
Evolutionary amplification of a pseudogene
}

\author{
BY P. GHAZAL, J. CLARK AND J. O. BISHOP \\ Department of Genetics, University of Edinburgh, West Mains Road, \\ Edinburgh EH9 3JN
}

The multigene family of the BALB/c mouse Major Urinary Proteins (MUPs) is comprised mainly of two homogenous groups of genes (Group 1 and Group 2 genes) each with about 15 members which are clustered on chromosome 4 . The unit of the MUP gene cluster $(\sim 750 \mathrm{~Kb})$ is a $45 \mathrm{~Kb}$ region which contains the head to head linkage of a Group 1 and Group 2 gene. Sequence analysis of a number of Group 1 and Group 2 genes has shown the Group 2 genes to be possible pseudogenes which share a common lesion. It is, therefore, suggested that the ancestral Group 2 pseudogene has been co-amplified along with the functional Group 1 gene.

\section{Construction of a shuttle vector based mutation system for use in mammalian cells}

\author{
By G. R. MacGREGOR, C. F. ARLETT and J. F. BURKE \\ MRC Cell Mutation Unit, University of Sussex, Falmer, Brighton
}

An attempt has been made to construct a shuttle vector based mutation system for use in mammalian cells. Two systems have been designed and feasibility of use of each for a mutation assay determined.

One system is based upon a $p B R:: S V 40$ construct in monkey COS-7 cells, the other a Co1E1: : Bovine Papilloma Virus (BPV) vector in murine C127 cells. Both vectors employ a cloned region of the $E$. coli lacZ gene as a target site for mutagenic events.

Data will be presented of the relative stability of each vector in its host system.

\section{Cloning of UDP-glucuronyltransferase cDNA}

\author{
BY MICHAEL R. JACKSON AND BRIAN BURCHELL
}

Biochemistry Department, Medical Sciences Institute, University of Dundee, Dundee DD1 $4 H N$

Hepatic microsomal UDP-glucuronyltransferase (GTs) are a family of isoenzymes involved in the facilitated elimination of potentially toxic endogenous compounds such as bilirubin and steroids as well as a wide range of xenobiotics. The isolation of genes coding for GT is a major step towards investigation of the heterogeneity, differential induction and genetic deficiencies of these enzymes.

Radioiodinated affinity purified anti GT antibodies have been used to isolate 
cloned GT cDNA for the first time. Screening of a rat liver cDNA library prepared in the expression vector $\lambda$ gt11 identified ten positive clones containing GT cDNA inserts ranging from $0 \cdot 3-2 \cdot 1 \mathrm{~Kb}$. The identity of the isolated cDNA was confirmed by hybrid select translation and immunochemical analyses.

Restriction mapping indicates that two classes of cDNA coding for different GT mRNA have been cloned.

\title{
Hypervariable regions in human DNA
}

\author{
BY ALEC J. JEFFREYS AND VICTORIA WILSON \\ Department of Genetics, University of Leicester, University Road, Leicester \\ $L E 17 R H$
}

The human genome contains large numbers of dispersed tandem-repetitive regions termed 'minisatellites' which share a common 10-15 bp core sequence reminiscent of the generalized recombination signal (chi) in $E$. coli. Probes have been constructed both for specific minisatellites and for the simultaneous detection of whole sets of minisatellites, and have been used to show that many of these regions are extremely polymorphic to the extent that they provide individualspecific DNA fingerprints. These minisatellites are dispersed in the genome, are mainly found on autosomes, and provide powerful genetic markers for pedigree analysis, inbreeding estimation, parenthood testing, etc.

\section{Immunization against rabies using a recombinant vaccinia virus expressing the rabies glycoprotein}

\author{
BY R. LATHE, M. P. KIENY, R. DRILLIEN, J. P. LECOCQ, \\ T. J. WIKTOR*, R. I. MACFARLAN* AND H. KOPROWSKI* \\ Transgene, S.A. and Institute of Virology, Strasbourg, France, and \\ *Wistar Institute, Philadelphia, U.S.A.
}

Vaccinia virus has been widely used to control and eradicate smallpox. VV has recently been developed as a vector to clone and express exogenous protein-coding sequences and we have adapted this system to the expression of the rabies surface glycoprotein.

The upstream G/C tail of the glycoprotein cDNA was removed and a cloning variant registering a leu-pro substitution at position 8 of the mature protein corrected by localised mutagenesis. This cDNA was introduced downstream of the VV 7.5 $K$ gene promoter and integrated into the viral $T K$ gene to create a selectable TK-deficient virus, VVTGgRAB-26D3.

Infection of cultured cells with the hybrid virus results in efficient expression of the rabies antigen. In vivo, the virus elicits high titres of rabies-neutralising antibodies and confers protection against severe challenge with street rabies virus. 


\title{
Possible paralogous chromosomal regions in mouse and man
}

\author{
BY LARS G. LUNDIN \\ Department of Medical and Physiological Chemistry, BMC, Box 575, \\ S-75123 Uppsala, Sweden
}

Three groups of paralogous regions, conserved after tetraploidization, are suggested to be carried on the following chromosomes:

\begin{tabular}{lrrrrrrrrr} 
& \multicolumn{4}{c}{ Mouse } & \multicolumn{5}{c}{ Man } \\
I & 1 & 6 & 7 & 9 & 11 & 12 & 15 & 19 & - \\
II & 2 & 4 & 17 & $X$ & $1 \mathrm{p}$ & 6 & 9 & 22 & $X$ \\
III & - & 8 & 19 & - & - & 10 & 16 & - & -
\end{tabular}

The first group carries, among others, genes for isomerases, peptidases, dehydrogenases, cytochromes and apolipoproteins. The second group carries many genes related to the immune system. The third group is, so far, small, and includes transaminase, lipase and esterase/protease genes. An interesting case of possible paralogy is the Ren-1 and -2 genes on mouse chr. 1 and the Prt-4,5-Tam-1 cluster on chr. 7.

\section{Variation in histidine decarboxylase levels and the response to hormones in mouse kidney}

\section{By R. J. MIDDLETON, S. A. M. MARTIN AND GRAHAME BULFIELD}

Genetics Group, AFRC's Poultry Research Centre, Roslin, Midlothian EH25 9PS, U.K. and Department of Genetics, University of Edinburgh, EH9 3JN, U.K.

Modulation of histidine decarboxylase (HDC) in the mouse kidney is affected by at least three hormones. Levels are induced by thyroxine and to a lesser extent by oestradiol, and repressed by testosterone. Amongst inbred and wild strains of mice variation exists in response to these hormones. This can be exhibited by lack of sex difference-normally females show higher HDC activity than males. Physiological measurements have been made on one of these strains (DAN) and the genetics of system has been examined. Reduced inducibility by thyroxine has been found in the strain SWR and total thyroxine insensitivity has been discovered in a strain of Mus spretus. Mutant androgen receptor $(T f m)$ leads to superinducibility of HDC by testosterone. A mechanism for this phenomenon will be discussed. 


\title{
Genetic variation for enzyme structure and systemic regulation in two haplotypes of the $\beta$-glucuronidase gene of $M$. m. castaneus
}

\author{
By K. PFISTER, G. WATSON*, AND K. PAIGEN* \\ University of Zurich, Switzerland, and *University of California, Berkeley, U.S.A.
}

The new haplotypes of the (Gus) gene complex have been characterized following their transfer from $M . m$. castaneus to a $\mathrm{C67BL} / 6 \mathrm{~J}$ genetic background. The $(G u s)^{C S}$ haplotype carries a new structural allele coding for enzyme with decreased thermolability and lacking an antigenic site present in other $\beta$-glucuronidase allozymes. The $(G u s)^{C L}$ haplotype carries another new structural allele coding for enzyme with increased thermolability and possessing the antigenic site. Both CS and CL $\beta$-glucuronidase have the same molecular activity as the standard B allozyme from C57BL/6J mice. Mice carrying either the $(G u s)^{C S}$ or the $(G u s)^{C L}$ haplotype have reduced enzyme activity in all tissues examined at all stages of development. The reduced enzyme activity is partially accounted for by reduced rates of enzyme synthesis; the remainder probably results from increased rates of enzyme turnover. $\beta$-Glucuronidase $\mathrm{mRNA}$ levels in these mice were not reduced suggesting that the observed reduction in enzyme synthesis is due to a decreased efficiency of mRNA translation.

\section{Comparative studies of the cytoplasmic protein from experimentally induced trisomic mice (TS19) and disomic controls from the same litter}

\author{
BY GERD H. REICHERT AND OLAF-GEORG ISSINGER
}

Institut fur Humangenetik, Univ. d. Saarlandes, D-6650 Homburg-3, FRG

Whole mouse embryos were homogenized and the crude cell lysate was fractionated by differential centrifugation into the various sub-cellular components (nuclei, membranes, mitochondria, polyribosomes, cytoplasmic proteins). The cytoplasmic protein fraction was further analysed by DEAE-Sepharose chromatography. Characterization of the column fractions by isoelectrofocusing 2D PAGE, was followed by silver-staining of the gels, leading to the identification of 14 quantitative and qualitative differences in the cytoplasmic protein pattern between trisomic and disomic controls. The prefractionation of the crude cell lysate before 2D PAGE analysis, reduced the amount of protein spots obtained per gel from 800 to 250. This then enabled us to compare differences in the protein pattern of trisomic and disomic controls. Five of the 14 observed differences in the protein pattern were due to newly synthesized protein present in the trisomic tissue and 6 proteins were found only in the disomic controls. 


\title{
Molecular analysis at the hypoxanthine phosphoribosyl-transferase (HPRT) locus
}

\author{
BY BELINDA ROSSITER \\ Biochemical Genetics, Paterson Laboratories, Christie Hospital and Holt Radium \\ Institute, Manchester M20 $9 B X$
}

Three spontaneous 6-thioguanine ${ }^{\mathrm{R}}$ mutants derived from V79S wild-type Chinese hamster cells have been studied. Each reverts to a $\mathrm{HAT}^{\mathrm{R}}$ phenotype on treatment with monofunctional alkylating agents, but not other mutagens. One (V79STG15) possesses a HPRT mRNA of wild-type level and size as shown by Northern analysis. The other 2 have no detectable HPRT sequences in mRNA nor in total RNA. Southern analysis following digestion of DNA from the 3 mutant lines with 8 different restriction enzymes showed no detectable difference between the wild-type and mutant HPRT sequences. The V79STG15 line may have a nonsense or missense mutation within the HPRT coding sequence and it is intended to compare the sequence of V79STG15 HPRT cDNA with that of the Chinese hamster wild-type, which is already known. A bacterial cDNA library from V79STG15 mRNA has been generated and 40000 recombinants have been screened for HPRT sequences but none was found. Genomic libraries of the other 2 mutant lines will be constructed and the $5^{\prime}$ end of the HPRT genes sequenced and compared with that of the wild-type, since the original mutation may be within the control region of the HPRT gene.

\section{Characterization of $\mathrm{CHO}$ cell lines resistant to antimicrotubular $-=-$ drugs}

\author{
BY J. R. WARR, K. ADAMS, F. BREWER AND M. A. ANDERSON \\ Department of Biology, University of York, York YO1 5DD
}

CHO cell lines resistant to vincristine, colchicine, taxol and other antimicrotubular drugs have been isolated. Selection has been either by single challenge to a high drug concentration or by prolonged exposure to gradually increasing drug concentrations. In the former case, the mutants have been analysed for complementation in cell hybrids and for gene location by microcell fusion. In the latter case, the strains are being analysed for gene amplification. In both cases, the phenotypes are being characterized in terms of drug uptake studies and 2D-gel electrophoresis of mutant proteins. 


\title{
Additional polymorphic systems in the domestic cat, Felis catus
}

\author{
BY A. VAN DE WEGHE, Y. BOUQUET, D. MATTHEEUWS*, A. VAN \\ ZEVEREN AND H. VAREWYCK
}

Department of Animal Genetics and Breeding, ${ }^{*}$ Clinic for Small Animals, Faculty of Veterinary Science, State University of Ghent, Heidestraat, 199220 Merelbeke, Belgium

Genetic variation was found in serum and erythrocytes of domestic cats by 2-dimensional non-denaturing electrophoresis. Family studies indicate that 6 serum polymorphic systems and a red cell esterase are controlled by different codominant autosomal alleles.

One of the serum systems has been identified as the Gc, whereas a second system showed a specific anti-trypsin activity. Additional variant chymotrypsin inhibiting forms have also been observed.

This study confirms that the variation, observed at these loci, is under genetic control. 